\title{
Recidiva do apinhamento anterossuperior nas más oclusões de Classe I e Classe II tratadas ortodonticamente sem extrações
}

\author{
Willian J. G. Guirro*, Karina Maria Salvatore de Freitas**, Marcos Roberto de Freitas ${ }^{\star \star}$,

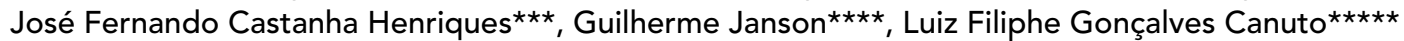

\section{Resumo}

Objetivo: o presente estudo objetivou comparar retrospectivamente a estabilidade pós-contenção do alinhamento dos incisivos anterossuperiores em pacientes com Classe I e Classe II. Métodos: a amostra consistiu de 38 pacientes de ambos os sexos, tratados sem extrações e com mecânica Edgewise, divididos em dois grupos - Grupo 1, constituído por 19 pacientes, com idade inicial média de 13,06 anos, portadores da má oclusão de Classe I com apinhamento anterossuperior inicial maior que 3mm; Grupo 2, constituído por 19 pacientes, com idade inicial de 12,54 anos, portadores da má oclusão de Classe II e, também, com apinhamento anterossuperior inicial maior que $3 \mathrm{~mm}$. Foram medidos nos modelos de estudo, das fases pré- e pós-tratamento e pós-contenção, o índice de irregularidade de Little, as distâncias intercaninos e entre os primeiros e segundos pré-molares, a distância intermolares e o comprimento da arcada superior. Para a comparação intragrupo nos 3 tempos de avaliação, utilizou-se os testes ANOVA e Tukey. A comparação intergrupos foi realizada pelo teste $t$ independente. Para verificação da presença de correlação, utilizou-se o teste de correlação de Pearson. Resultados: os resultados evidenciaram maior estabilidade do tratamento no Grupo 2 (Classe II), pois, durante o período pós-contenção, foi observada recidiva do apinhamento dos dentes anterossuperiores menor no Grupo 2 (0,80 mm) do que no Grupo 1 $(1,67 \mathrm{~mm})$. Conclusão: concluiu-se que o tratamento do apinhamento dos dentes anterossuperiores é mais estável na má oclusão de Classe II do que na má oclusão de Classe I.

Palavras-chave: Recidiva. Apinhamento anterossuperior. Estabilidade pós-tratamento.

\section{INTRODUÇÃO}

Os tratamentos ortodônticos têm como objetivo primordial a correção das más oclusões, entretanto, essas correções apresentam considerável Como citar este artigo: Guirro WJG, Freitas KMS, Freitas MR, Henriques
JFC, Janson G, Canuto LFG. Recidiva do apinhamento anterossuperior nas
más oclusões de Classe I e Classe II tratadas ortodonticamente sem extrações. Dental Press J Orthod. 2011 Sept-Oct;16(5):43.e1-16. variabilidade em relação à estabilidade pós-contenção. Embora exista um consenso na literatura ortodôntica de que algumas alterações oclusais irão ocorrer inevitavelmente após o término do

* Mestre em Ortodontia pela Faculdade de Odontologia de Bauru, Universidade de São Paulo.

** Mestre e Doutora em Ortodontia pela Faculdade de Odontologia de Bauru, Universidade de São Paulo. Coordenadora do Mestrado em Odontologia, área de concentração Ortodontia, da Faculdade Ingá, Maringá/PR.

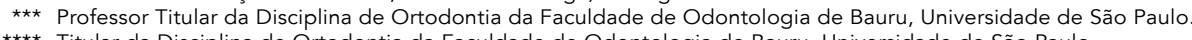

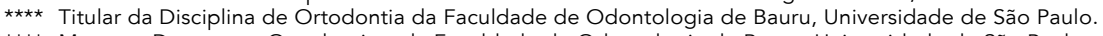

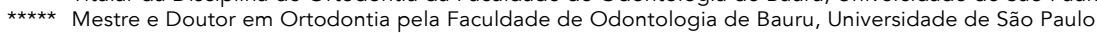


tratamento ortodôntico ${ }^{19,30}$, evidenciou-se que a estabilidade do alinhamento dos dentes é altamente variável e amplamente imprevisível ${ }^{2,20}$. Em relação à abordagem científica, apesar de numerosas pesquisas sobre a etiologia da recidiva no tratamento ortodôntico do apinhamento anteroinferior $^{3,4,11,18}$, uma menor quantidade de estudos foi conduzida com foco nas alterações pós-tratamento na região anterossuperior e nos possíveis fatores associados à magnitude dessa recidiva ${ }^{9,14,23,27,29}$.

\section{REVISÃO DA LITERATURA}

Evidenciou-se, após várias décadas de estudos, que a estabilidade do alinhamento dos dentes apresenta grande variabilidade entre os pacientes ${ }^{11}$. Little ${ }^{17}$ observou que casos tratados com ou sem extrações, assim como casos que não sofreram intervenção ortodôntica, apresentaram redução do perímetro e comprimento da arcada, achados que concordam com relatos de outros pesquisadores ${ }^{20,28,32}$. Citou, ainda, que nenhuma variável - seja ela de achados clínicos, modelos de estudo ou radiográficos antes ou após o tratamento - parece prever efetivamente a recidiva. Entretanto, assim como na maioria dos estudos relacionados à recidiva do apinhamento na região anterior, tais achados foram descritos somente para a arcada inferior. Portanto, a extrapolação dessas conclusões para a arcada superior deve ser feita com restrições.

A recidiva do apinhamento anterossuperior apresenta menor prevalência quando comparada à recidiva na região dos incisivos inferiores ${ }^{26,31,32}$.

A estabilidade do tratamento é um dos objetivos mais importantes da Ortodontia, porém, apesar de décadas de estudos, há um consenso de que a estabilidade na correção do apinhamento é amplamente variável e imprevisível ${ }^{11}$. A ocorrência de recidiva do apinhamento na arcada superior é de aproximadamente $7 \%$ no período pós-contenção ${ }^{29}$, no entanto, existem evidências de que o índice de irregularidade tende a recidivar em 23\% dos casos ${ }^{15}$.

Para Little ${ }^{16}$, a evidência da progressiva estabilidade do tratamento ortodôntico é sempre primeiramente notada pelo apinhamento dos incisivos in- feriores após a remoção da contenção. Independentemente do fator oclusal da recidiva ortodôntica, a irregularidade dos incisivos inferiores apresenta-se sempre como a precursora do apinhamento superior. Além disso, a recidiva do apinhamento anteroinferior pode estar relacionada com o aprofundamento da sobremordida, assim como a deterioração generalizada do caso tratado ${ }^{16}$.

Sadowsky et al. ${ }^{27}$, em 1994, realizaram um estudo avaliando casos ortodônticos previamente tratados, para avaliar a estabilidade em longo prazo do alinhamento dos incisivos superiores e inferiores. Todos os casos foram tratados sem extrações e utilizando-se o aparelho tipo Edgewise, sendo avaliados no período mínimo de 5 anos sem contenção. Os dados foram obtidos dos modelos do estudo. O tempo médio de uso da contenção inferior fixa foi de 8,4 anos. $\mathrm{O}$ índice de irregularidade antes do tratamento era de, em média, $8,0 \mathrm{~mm}$ na maxila e $5,2 \mathrm{~mm}$ na mandibula; no fim do tratamento, $0,9 \mathrm{~mm}$ e $1,0 \mathrm{~mm}$, respectivamente; e, no estágio pós-contenção, 2,0mm e 2,4mm, respectivamente. O tratamento foi feito sem avanço dos incisivos ou distalização dos molares, entretanto, ambas as arcadas foram expandidas transversalmente. Durante o período pós-contenção, todas as variáveis mostraram uma recidiva estatisticamente significativa, exceto a distância intercaninos e interpré-molares na arcada superior expandida.

Vaden et al. ${ }^{32}$, em 1997, avaliaram as alterações na irregularidade dos incisivos superiores e inferiores e nas dimensões das arcadas dentárias entre os 6 e os 15 anos de idade, após a remoção das contenções. A amostra consistiu de 36 pacientes tratados com extrações. Observaram que tanto a arcada superior quanto a inferior apresentaram alguma redução dimensional durante o período pós-contenção. Após 15 anos do término do tratamento, os resultados mostraram um aumento de apenas $0,3 \mathrm{~mm}$ na irregularidade dos incisivos superiores, que correspondeu a uma manutenção de $96 \%$ da correção obtida durante o tratamento. De forma geral, 90\% dos pacientes apresentaram condições oclusais no período pós-contenção melhores do que as iniciais. 
Surbeck et al. ${ }^{29}$, em 1998, avaliaram os modelos de estudo de 745 pacientes ortodônticos e estudaram o alinhamento dos dentes anterossuperiores nos estágios pré-, pós-tratamento e pós-contenção, buscando verificar a influência da quantidade do apinhamento inicial na recidiva pós-contenção. Utilizaram como critério de seleção da amostra a presença de todos os dentes anterossuperiores em casos de pacientes tratados ortodonticamente com ou sem a realização de extrações dentárias. A amostra foi divida em três grupos, de acordo com a configuração dos modelos de estudo pós-contenção: (1) com espaços interdentários significativos; (2) com significativa recidiva do apinhamento; (3) com perfeito alinhamento dos dentes anterossuperiores. Após a análise dos resultados, os autores observaram que a quantidade de irregularidade no período pós-contenção apresentou-se diretamente relacionada com a quantidade de deslocamento dos pontos de contato anatômicos no início do tratamento, e que dentes rotacionados na fase pré-tratamento apresentaram uma tendência estatisticamente significativa de retornar às suas posições iniciais.

Huang e Artun $^{14}$, em 2001, avaliaram uma possível associação entre a irregularidade no alinhamento dos incisivos superiores e a recidiva do apinhamento anterossuperior. Sugeriram que o contato dos dentes anterossuperiores com os dentes inferiores limitaria os movimentos dos dentes superiores. Dessa forma, as alterações no posicionamento dos dentes superiores são, geralmente, resultantes de forças exercidas pelos lábios. De acordo com os autores, o posicionamento dos incisivos inferiores e a função labial poderiam ter maior importância na recidiva no sentido vestibulolingual dos dentes anterossuperiores do que os movimentos realizados durante a mecânica ortodôntica. Sugeriram que o posicionamento dos dentes anteroinferiores influencia no posicionamento dos dentes superiores e vice-versa e, dessa forma, a recidiva da região anterior em uma das arcadas poderia estar associada à recidiva do alinhamento dos dentes da arcada oposta. A amostra avaliada consistia de 96 pacien- tes, apresentando, na maioria dos casos, má oclusão de Classe II tratada com ou sem extrações dentárias. O período pós-contenção variou de 4 a 25 anos. Os resultados demonstraram uma associação estatisticamente significativa entre a irregularidade no alinhamento dos dentes anterossuperiores e a recidiva do alinhamento dos dentes da arcada inferior nessa mesma região, assim como uma associação entre o deslocamento dos dentes no sentido vestibulolingual nos segmentos anteriores superior e inferior.

Naraghi et al. ${ }^{24}$, em 2006, realizaram um estudo para avaliar a estabilidade pós-tratamento do alinhamento de incisivos superiores que receberam uma contenção fixa após o tratamento. Foram observados 135 modelos de estudo de 45 pacientes, nos períodos pré-tratamento ( $\mathrm{Tl}$ ), no ato da colagem da contenção (T2) e 1 ano após a remoção das contenções (T3). Todos os pacientes foram tratados com aparelhos fixos do tipo Edgewise, e o índice de irregularidade dos incisivos foi calculado nos três grupos. A irregularidade média dos incisivos no grupo $\mathrm{T} 1$ era de $10,1 \mathrm{~mm}$, no $\mathrm{T} 2$ era de $0,7 \mathrm{~mm}$ e no T3 era de $1,4 \mathrm{~mm}$. Cinquenta e cinco dentes de 42 pacientes foram corrigidos em mais de $20^{\circ}$ de rotação entre as fases $\mathrm{T} 1$ e $\mathrm{T} 2$ e a recidiva média dessa correção foi de $7,3^{\circ}$. O ponto de contato entre os incisivos centrais e laterais foi o ponto mais crítico perante o nível de recidiva. Após 1 ano de remoção das contenções, observou-se uma mínima, ou nenhuma, recidiva do apinhamento dos dentes anterossuperiores submetidos à contenção fixa.

Erdinc, Nanda e Isiksal ${ }^{8}$, em 2006, avaliaram 98 pacientes, de ambos os sexos, que apresentavam má oclusão de Classe I e de Classe II, divisão 1 de Angle. Os pacientes foram avaliados nas fases pré-tratamento, final do tratamento ativo e pós-contenção. A amostra foi dividida em dois grupos (49 indivíduos cada), tratados sem extrações ou com extrações dos quatro primeiros pré-molares. Não houve crescimento estatisticamente significativo em nenhum dos grupos no período avaliado. Após a análise de modelos e cefalométrica, observou-se que a única medida com diferença estatisticamente significativa 
entre os grupos foi o ângulo interincisivos. Ambos os grupos mostraram uma melhora significativa na sobressaliência e não houve recidiva considerável. Houve, também, uma melhora na sobremordida em ambos os grupos, sendo que o grupo tratado sem extrações apresentou melhores resultados; porém, foi observada uma recidiva significativa nos dois grupos. O tratamento produziu uma melhora estatisticamente significativa no apinhamento dos incisivos, tanto superiores quanto inferiores, em ambos os grupos, e os incisivos inferiores apresentaram uma recidiva considerável desse apinhamento, sendo, respectivamente, de $0,97 \mathrm{~mm}$ e $0,99 \mathrm{~mm}$ nos grupos tratados com e sem extrações. A recidiva do apinhamento anterossuperior apresentou-se menor do que a recidiva do apinhamento anteroinferior entre o final do tratamento ativo e o período pós-contenção; porém, essas alterações não foram significativas. $\mathrm{O}$ alinhamento superior permaneceu estável, mas o apinhamento anteroinferior recidivou em média lmm, em ambos os grupos. De acordo com Little ${ }^{16}$, essa recidiva é considerada mínima. Sendo assim, o presente estudo mostrou uma ótima estabilidade pós-contenção do alinhamento dos incisivos superiores e inferiores, pois o mínimo de apinhamento dos incisivos ocorreu no período pós-contenção e a recidiva foi semelhante nos grupos tratados com e sem extrações de primeiros pré-molares.

Canuto $^{5}$, em 2006, apresentou um estudo que objetivou comparar, por meio de uma análise retrospectiva, a estabilidade pós-contenção do alinhamento dos incisivos anterossuperiores de pacientes submetidos ou não ao procedimento de expansão rápida da maxila durante o tratamento ortodôntico. A amostra consistiu-se de 48 pacientes de ambos os sexos, apresentando más oclusões de Classes I e II, tratados sem extrações e com mecânica Edgewise. A amostra total foi dividida em dois grupos, de acordo com o protocolo de tratamento: Grupo 1 (com ERM) constituído por 25 pacientes, com idade inicial média de 13,53 anos, submetidos à expansão rápida da maxila durante o tratamento ortodôntico. Grupo 2 (sem ERM) apresentando
23 pacientes, com idade inicial de 13,36 anos, cujo tratamento ortodôntico não priorizou a expansão transversal maxilar. Foram medidos — nos modelos de estudo das fases pré- (T1), pós-tratamento (T2) e pós-contenção (T3) — o índice de irregularidade de Little, as distâncias intercaninos e entre os primeiros e segundos pré-molares, a distância intermolares, o comprimento e o perímetro da arcada superior. Os resultados evidenciaram incrementos dimensionais transversais significativamente maiores no grupo tratado com ERM (Grupo 1). Entretanto, durante o período pós-contenção, não foram observadas diferenças significativas entre os grupos em relação à quantidade de aumento na irregularidade dos incisivos superiores $(+1,52 \mathrm{~mm}$ em ambos os grupos), bem como em relação à maioria das variáveis estudadas. Concluiu-se que a realização do procedimento de expansão rápida da maxila não apresentou influência na recidiva do apinhamento anterossuperior em longo prazo.

Martins $^{21}$, em 2007, apresentou um estudo que objetivou avaliar, retrospectivamente, a influência da expansão rápida da maxila na estabilidade pós-contenção do alinhamento dos dentes anterossuperiores em casos tratados com extração de pré-molares. A amostra foi constituída de 60 pacientes de ambos os sexos, com más oclusões de Classe I e de Classe II, tratados com extrações de quatro pré-molares, utilizando-se a mecânica de Edgewise. A amostra foi dividida em dois grupos, de acordo com o protocolo de tratamento, sendo o Grupo 1 composto por 30 pacientes, com idade inicial média de 13,55 anos, tratados ortodonticamente com extrações de quatro pré-molares. O Grupo 2 apresentou 30 pacientes, com idade inicial média de 13,98 anos tratados ortodonticamente com expansão rápida da maxila e, posteriormente, mecânica corretiva com extrações de quatro pré-molares ou dois pré-molares superiores. Foram avaliados os modelos de estudo referentes às fases inicial (T1), final (T2) e pós-contenção (T3) de cada paciente, medindo-se o índice de Little, as distâncias intercaninos, interpré-molares, intermolares, o comprimento e o perímetro da arcada. 
Os resultados evidenciaram que o índice de irregularidade de Little apresentou $9,40 \%$ de recidiva para o Grupo 1 e 13,57\% para o Grupo 2. Não ocorreu diferença estatisticamente significativa entre os grupos na recidiva das distâncias intercaninos, interpré-molares e intermolares, no comprimento e no perímetro da arcada. Entretanto, o Grupo 2 apresentou maior quantidade de recidiva na quantidade de apinhamento anterossuperior em relação ao Grupo 1. Portanto, houve influência da expansão rápida da maxila na estabilidade do alinhamento dos incisivos superiores.

\section{PROPOSIÇÃO}

O objetivo desse estudo retrospectivo foi avaliar a recidiva do apinhamento anterossuperior em casos tratados ortodonticamente sem extrações, utilizando o índice de irregularidade de Little, para:

» Comparar a recidiva do apinhamento anterossuperior entre as más oclusões de Classe I e de Classe II de Angle.

" Correlacionar o índice de irregularidade de Little, as distâncias intercaninos, interpré-molares e intermolares, assim como o comprimento da arcada superior ao início e ao final do tratamento e no período pós-contenção.

\section{MATERIAL E MÉTODOS \\ Material}

A amostra utilizada nesse estudo retrospectivo constitui-se de 38 documentações ortodônticas de pacientes tratados no curso de Pós-Graduação em Ortodontia da Universidade de São Paulo / Faculdade de Odontologia de Bauru, os quais apresentavam, inicialmente, más oclusões de Classe I ou de Classe II, tratados ortodonticamente sem extrações. Os critérios para a seleção da amostra também incluíram a presença de todos os dentes permanentes irrompidos ao início do tratamento ortodôntico (até os primeiros molares) e a ausência de anomalias dentárias de forma e/ou número. Todos os pacientes foram tratados com aparelhos fixos e mecânica Edgewise e apresentavam docu- mentação ortodôntica completa, incluindo os modelos de estudo das fases inicial, final de tratamento e de controle pós-contenção.

A amostra foi dividida em dois grupos, conforme a classificação da má oclusão segundo Angle. Dessa forma, os grupos foram assim distribuídos:

» Grupo 1 (pacientes Classe I de Angle): constituído por 19 pacientes que apresentavam apinhamento anterossuperior ao início do tratamento ortodôntico.

»Grupo 2 (pacientes Classe II de Angle): constituído por 19 pacientes que apresentavam apinhamento anterossuperior ao início do tratamento ortodôntico.

Todos os pacientes utilizaram como contenção, ao final do tratamento ortodôntico ativo, uma placa de Hawley removível na arcada superior e um 3x3 colado de canino a canino na arcada inferior. A placa de contenção superior foi utilizada, em média, por 1 ano, enquanto o $3 \times 3$ permaneceu por um período médio de 3 anos.

\section{Grupo 1 (Classe I de Angle)}

O grupo Classe I de Angle (Grupo 1) apresentou 19 pacientes leucodermas (12 do sexo feminino e 7 do sexo masculino), com idade inicial média de 13,06 anos $( \pm 1,27)$. O tempo médio de tratamento ortodôntico corretivo foi de 2,15 anos $( \pm 0,89)$. Ao término do tratamento, todos os pacientes apresentavam uma finalização satisfatória. Nessa fase final, os pacientes apresentavam uma média de idades de 15,19 anos $( \pm 1,24)$. Os pacientes pertencentes ao Grupo 1 foram reavaliados após um período pós-contenção médio de 8,60 anos $( \pm 1,83)$.

Em relação à má oclusão inicial, o Grupo 1 apresentou 19 pacientes com má oclusão de Classe I, apresentando uma irregularidade anterossuperior, segundo Little ${ }^{16}$, maior ou igual a $3 \mathrm{~mm}$.

\section{Grupo 2 (Classe II de Angle)}

O Grupo 2, constituído por pacientes que inicialmente apresentavam má oclusão de Classe II 
de Angle, apresentou 19 pacientes leucodermas (14 do sexo feminino e 5 do sexo masculino), com idade média de 12,54 anos $( \pm 1,37)$ ao início da terapia ortodôntica. $\mathrm{O}$ tempo médio de tratamento foi de 2,32 anos $( \pm 0,73)$. Ao término do tratamento, todos os pacientes, assim como os pacientes pertencentes ao Grupo 1, apresentavam uma finalização satisfatória. Nessa fase, os pacientes apresentavam uma média de idades de 14,93 anos $( \pm 1,50)$ e foram reavaliados após um período pós-contenção médio de 8,04 anos $( \pm 2,11)$.

Em relação à má oclusão inicial, o Grupo 2 apresentou 19 pacientes com má oclusão de Classe I, apresentando uma irregularidade anterossuperior, segundo Little ${ }^{16}$, maior ou igual a $3 \mathrm{~mm}$.

\section{Métodos}

Nos arquivos do Departamento de Odontopediatria, Ortodontia e Saúde Coletiva da Faculdade de Odontologia de Bauru (FOB) / Universidade de São Paulo, Disciplina de Ortodontia, foram examinadas todas as pastas ortodônticas e os modelos de gesso iniciais, finais e de avaliação pós-contenção, no intuito de selecionar a amostra seguindo os critérios supracitados. Todos esses pacientes haviam sido tratados por alunos de pós-graduação, nos cursos de especialização (Lato sensu) e mestrado/ doutorado (Stricto sensu) em Ortodontia, da referida instituição de ensino. Após o registro de todos os casos selecionados, descartaram-se aqueles cujos modelos apresentavam-se com artefatos de técnica, ausentes para uma ou mais fases avaliadas (inicial, final e pós-contenção), ou mesmo bastante danificados, a ponto de impossibilitar seu uso.

As pastas ortodônticas relativas à amostra selecionada foram utilizadas para a obtenção de alguns dados relevantes à condução dessa pesquisa. A ficha de dados cadastrais foi utilizada para o registro do nome completo dos pacientes, o sexo e a data de nascimento. As fichas de procedimentos terapêuticos foram examinadas quanto às datas de início e término do tratamento e de realização dos controles pós-tratamento. Também se observou a data de remoção das contenções. Esses dados, em conjunto com a data de nascimento do paciente, permitiram a determinação exata do tempo total de tratamento, do tempo de controle pós-tratamento e da idade dos pacientes nas fases estudadas.

Quando constatados fatores que prejudicariam a padronização da amostra - como, por exemplo, falhas na manutenção da documentação e dos modelos, incompatibilidades em relação à severidade e o tipo de má oclusão inicial ou tempo de avaliação pós-contenção inadequado - , o caso era imediatamente excluído da amostra.

\section{Avaliação dos modelos de estudo}

Os modelos de gesso das fases de início do tratamento ( $\mathrm{T} 1$, inicial), final de tratamento (T2, final) e pós-contenção (T3, pós-contenção) foram avaliados. Os modelos de avaliação pós-tratamento foram obtidos pelo menos 5 anos após o término do tratamento. Todas as medidas realizadas foram obtidas utilizando-se um paquímetro digital da marca Mitutoyo (Mitutoyo Sul Americana Ltda., São Paulo, Brasil, modelo/código 500143B), com capacidade de $150 \mathrm{~mm}$, com precisão de até $0,01 \mathrm{~mm}$.

As variáveis estudadas nos modelos de estudo superiores foram:

" Índice de irregularidade de Little (modificado) (LITTLE): o índice de irregularidade proposto por Little $^{16}$ foi idealmente elaborado para a avaliação do apinhamento dentário no segmento anteroinferior. Entretanto, devido à sua grande reprodutibilidade e precisão, a mesma metodologia para a avaliação do deslocamento dentário foi utilizada no presente estudo para a avaliação do apinhamento anterossuperior. $\mathrm{O}$ índice de Little foi calculado nos modelos de gesso superiores nas três fases estudadas (LITTLE1, LITTLE2 e LITTLE3). Para essa medição, utilizou-se um paquímetro digital, posicionado paralelamente ao plano oclusal. Aferiu-se, desse modo, o índice de irregularidade, que se caracterizou pelo somatório das distâncias lineares entre os pontos de contato anatômicos 
dos dentes anterossuperiores (caninos e incisivos). Essa medida representa a distância para a qual os pontos de contato devem ser movidos para atingir um alinhamento. De acordo com Little ${ }^{16}$, apesar dos pontos de contato poderem variar no sentido vertical, a correção dessas discrepâncias não afetará significativamente o comprimento anterior da arcada e, por isso, alterações no sentido vertical foram desconsideradas (Fig. 1).

" Distância intercaninos (INTERC): distância medida, em milímetros, entre as pontas das cúspides dos caninos superiores direito e esquerdo. Nos casos em que os caninos apresentavam facetas de desgaste, a ponta da cúspide foi estimada.

» Distâncias interpré-molares (INTERPB e INTERPB'): distâncias medidas, em milímetros, entre as fossas mesiais dos primeiros pré-molares superiores direito e esquerdo (B) e dos segundos pré-molares superiores direito e esquerdo (B'), respectivamente.

» Distância intermolares (INTERM): distância medida, em milímetros, entre as pontas das cúspides mesiovestibulares dos primeiros molares superiores direito e esquerdo. Nos casos em que os molares apresentavam facetas de desgaste, a ponta da cúspide foi estimada.

" Comprimento da arcada superior (COMPR): somatório das distâncias medidas entre o ponto de contato dos incisivos centrais superiores e a face mesial dos primeiros molares nos lados direito e esquerdo.

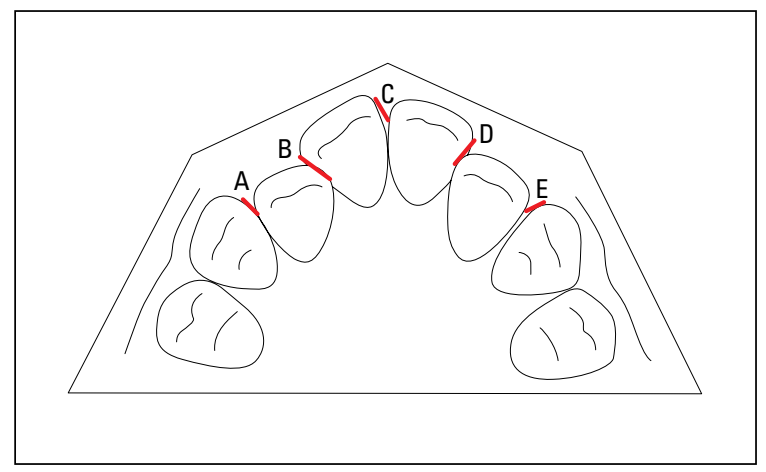

FIGURA 1 - Índice de irregularidade de Little $($ modificado $)=A+B+C+D+E$.

\section{Erro do método}

O erro intraexaminador foi avaliado realizando-se novas medidas das variáveis estudadas nos modelos iniciais, finais e pós-contenção de 10 pacientes, selecionados aleatoriamente, pertencentes a ambos os grupos. As variáveis reavaliadas (LITTLE, INTERC, INTERPB, INTERPB', INTERM e COMPR) também foram selecionadas de forma aleatória. A primeira e a segunda medições foram realizadas com intervalo de tempo de um mês. A fórmula proposta por Dahlberg ${ }^{6}\left(\mathrm{Se}^{2}=\Sigma \mathrm{d}^{2} / 2 \mathrm{n}\right)$ foi aplicada para estimar a ordem de grandeza dos erros casuais, enquanto os erros sistemáticos foram analisados pela aplicação de testes $t$ pareados, de acordo com Houston ${ }^{13}$.

\section{Método estatístico}

Foi realizada a estatística descritiva (média, desvio-padrão e número), para os Grupos 1 (Classe I) e 2 (Classe II), das medidas obtidas para as variáveis índice de irregularidade de Little, distância intercaninos, distâncias entre os primeiros e segundos pré-molares, distância intermolares e comprimento da arcada, nas fases inicial (T1), final (T2) e pós-contenção (T3). A estatística descritiva também foi realizada para a diferença das medidas obtidas a partir dos modelos entre as fases final e inicial (T21), caracterizando a correção com o tratamento; e para a diferença entre as fases pós-contenção e final (T3-T2), caracterizando a alteração durante o período pós-contenção; e para a diferença entre as fases pós-contenção e inicial (T3-T1), caracterizando as alterações no período total de observação.

Para avaliação da compatibilidade entre os grupos 1 e 2 quanto à distribuição dos sexos e em relação à severidade inicial da má oclusão, utilizou-se o teste qui-quadrado. Com o intuito de verificar a compatibilidade dos grupos quanto à quantidade de apinhamento inicial (LITTLE); idades inicial (Id T1), final (Id T2) e pós-contenção (Id T3); tempo de tratamento (TTRAT); tempo de contenção (TCONT) e de avaliação pós-contenção (TPOS), utilizou-se o teste $t$ independente. 
Para a comparação intragrupo nos três tempos de avaliação (T1, Inicial; T2, Final; T3, Pós-contenção), utilizou-se o teste ANOVA dependente e, em caso de resultado significativo, o teste de Tukey. O teste foi empregado para avaliação do comportamento das variáveis medidas nos modelos dos Grupos 1 e 2 conjuntamente, nos três tempos estudados.

Para a comparação intergrupos quanto aos valores obtidos para as variáveis avaliadas nos modelos nas fases inicial, final de tratamento e pós-contenção, assim como em relação às alterações dessas variáveis durante o tratamento (T2-T1), durante o período pós-contenção (T3-T2) e totais (T3-T1), utilizou-se o teste $t$ independente.

Por fim, utilizou-se o teste de correlação de Pearson para verificar a presença de correlação entre a recidiva do apinhamento anterossuperior e a recidiva das variáveis: distâncias intercaninos, interpré-molares, intermolares e comprimento da arcada.

Todos os testes foram realizados com o programa Statistica (Statistica for Windows - Release 6.0 - Copyright StatSoft, Inc. 2001), adotando-se um nível de significância de 5\%.

\section{RESULTADOS}

A Tabela 1 mostra os resultados da avaliação dos erros casuais e sistemáticos, realizados por meio da fórmula de Dahlberg 6 e de testes $t$ pareados, respectivamente, aplicados às variáveis LITTLE, INTERC, INTERPB, INTERPB', INTERM e COMPR, medidas nos modelos de estudo com intervalo de tempo de um mês.

A compatibilidade dos grupos quanto à proporção dos sexos foi avaliada pelo teste qui-quadrado (Tab. 2). Não houve diferença estatisticamente significativa entre os grupos em relação à distribuição dos sexos.

O teste $t$ independente foi utilizado para avaliação da compatibilidade dos grupos quanto à quantidade de apinhamento anterossuperior inicial (LITTLE1), quanto à idade inicial (Id T1), idade final (Id T2) e idade pós-contenção (Id T3)
TABELA 1 - Resultados do teste t e da fórmula de Dahlberg ${ }^{6}$, aplicados às variáveis avaliadas para estimativa dos erros sistemáticos $\mathrm{e}$ casuais, respectivamente.

\begin{tabular}{|c|c|c|c|c|c|c|}
\hline \multirow[t]{2}{*}{ Variáveis } & \multicolumn{2}{|c|}{$\begin{array}{c}1^{\text {a }} \text { medição } \\
(n=10)\end{array}$} & \multicolumn{2}{|c|}{$\begin{array}{c}2^{\mathrm{a}} \text { medição } \\
(\mathrm{n}=10)\end{array}$} & \multirow[t]{2}{*}{ Dahlberg } & \multirow[t]{2}{*}{$\mathbf{p}$} \\
\hline & média & d.p. & média & d.p. & & \\
\hline LITTLE1 & 7,02 & 3,08 & 7,05 & 3,13 & 0,05 & 0,397 \\
\hline LITTLE2 & 0,10 & 0,33 & 0,11 & 0,34 & 0,01 & 0,343 \\
\hline LITTLE3 & 1,47 & 0,81 & 1,49 & 0,80 & 0,02 & 0,422 \\
\hline INTERC1 & 34,21 & 3,06 & 33,25 & 4,58 & 0,25 & 0,322 \\
\hline INTERC2 & 34,38 & 2,28 & 34,44 & 2,25 & 0,07 & 0,135 \\
\hline INTERC3 & 34,60 & 2,33 & 34,57 & 2,34 & 0,05 & 0,527 \\
\hline INTERPB1 & 32,35 & 2,49 & 32,63 & 2,48 & 0,35 & 0,220 \\
\hline INTERPB2 & 36,03 & 2,09 & 35,82 & 2,23 & 0,26 & 0,246 \\
\hline INTERPB3 & 35,30 & 2,18 & 35,39 & 2,55 & 0,22 & 0,564 \\
\hline INTERPB' 1 & 37,04 & 3,41 & 37,10 & 3,64 & 0,28 & 0,755 \\
\hline INTERPB'2 & 41,13 & 2,56 & 41,21 & 2,59 & 0,15 & 0,454 \\
\hline INTERPB'3 & 40,40 & 2,39 & 40,48 & 2,64 & 0,20 & 0,564 \\
\hline INTERMOL1 & 49,21 & 3,94 & 49,29 & 3,86 & 0,10 & 0,207 \\
\hline INTERMOL2 & 52,19 & 3,85 & 52,33 & 3,80 & 0,13 & 0,092 \\
\hline INTERMOL3 & 52,38 & 3,50 & 52,48 & 3,71 & 0,15 & 0,303 \\
\hline COMPR1 & 67,96 & 5,22 & 68,21 & 5,51 & 0,25 & 0,121 \\
\hline COMPR2 & 72,59 & 4,59 & 72,58 & 4,75 & 0,23 & 0,990 \\
\hline COMPR3 & 70,86 & 4,06 & 71,36 & 4,71 & 0,65 & 0,249 \\
\hline
\end{tabular}

TABELA 2 - Resultados do teste qui-quadrado para avaliação da compatibilidade dos Grupos 1 e 2 quanto à proporção dos sexos.

$\begin{array}{ccccc} & \text { Feminino } & \text { Masculino } & \text { Total } \\ \text { Grupo 1 (Classe I) } & 12 & 7 & \mathbf{1 9} \\ \text { Grupo 2 (Classe II) } & 14 & 5 & \mathbf{1 9} \\ \text { Total } & \mathbf{2 6} & \mathbf{1 2} & \mathbf{3 8} \\ \mathrm{X}^{2}=0,49 & & \mathrm{df}=1 & & \mathrm{p}=0,485\end{array}$

TABELA 3 - Resultados do teste t independente, aplicado às variáveis índice de irregularidade de Little inicial; idade inicial, final e pós-contenção; tempo de tratamento e tempo de avaliação pós-contenção dos Grupos 1 e 2, para avaliação da compatibilidade intergrupos.

\begin{tabular}{cccccc} 
& \multicolumn{2}{c}{$\begin{array}{c}\text { Grupo } 1 \text { (Classe I) } \\
\text { Variáveis }\end{array}$} & \multicolumn{2}{c}{$\begin{array}{c}\text { Grupo 2 (Classe II) } \\
(\mathbf{n = 1 9 )}\end{array}$} & p \\
\cline { 2 - 4 } & média & d.p. & média & d.p. & \\
\cline { 2 - 4 } LITTLE1 & 7,83 & 3,14 & 6,35 & 2,67 & 0,126 \\
Id T1 & 13,06 & 1,27 & 12,54 & 1,37 & 0,233 \\
Id T2 & 15,19 & 1,24 & 14,93 & 1,50 & 0,552 \\
Id T3 & 21,67 & 2,52 & 20,62 & 2,41 & 0,201 \\
TTRAT & 2,15 & 0,89 & 2,32 & 0,73 & 0,534 \\
TPOS & 8,60 & 1,83 & 8,04 & 2,11 & 0,388
\end{tabular}


e quanto aos tempos de tratamento (TTRAT) e de avaliação pós-contenção (TPOS). Não foram observadas diferenças significativas entre os grupos em relação a essas variáveis (Tab. 3).

Os resultados do ANOVA para as variáveis medidas nos modelos de estudo dos Grupos 1 e 2, respectivamente, nas três fases estudadas $(\mathrm{T} 1, \mathrm{~T} 2 \mathrm{e}$ T3), encontram-se nas Tabelas 4 e 5 . Na presença de um resultado significativo, realizou-se o teste de Tukey, no qual letras diferentes demonstram diferença significativa entre as medições. Os resultados para o Grupo 1 demonstram uma significativa redução do apinhamento anterossuperior e incrementos dimensionais estatisticamente significativos entre as fases inicial (T1) e final (T2), com exceção da distância intercaninos (INTERC) (Tab. 4). Observa-se que essas alterações tenderam a se mostrar estáveis no período pós-contenção, com exceção da distância entre os primeiros pré-molares (INTER$\mathrm{PB}$ ) e o comprimento da arcada (COMPR), que tiveram uma diminuição estatisticamente significativa no período pós-contenção (T3) (Tab. 4). Os resultados do ANOVA para o Grupo 2 demonstram que o índice de Little sofreu alterações significativas durante o tratamento e durante o período de pós-contenção (Tab. 5). Entretanto, não houve alterações estatisticamente significativas para a maioria das demais variáveis estudadas durante essas fases,

TABELA 4 - Resultados do ANOVA para as variáveis dos modelos de estudo do Grupo 1 ( $n=19)$, nas três fases estudadas (T1, T2 e T3). Na presença de um resultado significativo, realizou-se o teste de Tukey (letras diferentes demonstram diferença significativa entre as medições).

\begin{tabular}{ccccc} 
& Inicial (T1) & Final (T2) & $\begin{array}{c}\text { Pós- } \\
\text { contenção (T3) }\end{array}$ & p \\
\cline { 2 - 4 } & média (d.p.) & média (d.p.) & média (d.p.) & \\
\cline { 2 - 4 } LITTLE & $7,83(3,14)^{\mathrm{A}}$ & $0,34(0,68)^{\mathrm{B}}$ & $2,01(1,87)^{\mathrm{B}}$ & $0,000^{*}$ \\
INTERC & $33,79(2,36)^{\mathrm{A}}$ & $34,46(1,48)^{\mathrm{A}}$ & $34,29(1,47)^{\mathrm{A}}$ & 0,306 \\
INTERPB & $32,62(1,91)^{\mathrm{A}}$ & $35,91(1,63)^{\mathrm{B}}$ & $34,66(1,54)^{\mathrm{C}}$ & $0,000^{*}$ \\
INTERPB' & $37,91(2,94)^{\mathrm{A}}$ & $40,90(2,19)^{\mathrm{B}}$ & $40,02(2,04)^{\mathrm{B}}$ & $0,000^{*}$ \\
\hline INTERMOL & $49,49(3,16)^{\mathrm{A}}$ & $51,53(2,86)^{\mathrm{B}}$ & $51,34(2,69)^{\mathrm{B}}$ & $0,000^{*}$ \\
\hline COMPR & $68,33(4,72)^{\mathrm{A}}$ & $71,01(3,45)^{\mathrm{B}}$ & $69,48(3,38)^{\mathrm{A}}$ & $0,000^{*}$
\end{tabular}

* Estatisticamente significativo para $\mathrm{p}<0,05$. com exceção das distâncias intercaninos (INTERC) e intermolares (INTERMOL), que sofreram uma diminuição significativa entre os tempos final (T2) e pós-contenção (T3) (Tab. 5).

As Tabelas 6, 7 e 8 mostram os resultados do teste $t$ independente para a comparação intergrupos das variáveis estudadas, nos tempos inicial (T1), final (T2) e pós-contenção (T3), respectivamente. $\mathrm{Na}$ fase inicial, não houve diferença estatisticamente significativa, para nenhuma das variáveis estudadas, entre os Grupos 1 e 2 (Tab. 6). Nas fases final e pós-contenção, foram observadas diferenças significativas entre os Grupos 1 e 2 para a quantidade de apinhamento anterossuperior (LITTLE2 e LITTLE3, Tab. 7 e 8, respectivamente).

As alterações das variáveis medidas nos modelos durante o tratamento (T2-T1), durante o período pós-contenção (T3-T2) e totais (T3-T1), em ambos os grupos, foram comparadas por meio de testes $\mathrm{t}$ (Tab. 9, 10 e 11, respectivamente). Em relação às alterações ocorridas durante o tratamento (T2-T1), não foram observadas diferenças significativas entre os Grupos 1 e 2 (Tab. 9). Durante o período pós-contenção (T3-T2), somente a quantidade de apinhamento anterossuperior apresentou diferença estatisticamente significativa entre os grupos (Tab. 10). Em relação às alterações totais (T3-T1), não foram

TABELA 5 - Resultados do ANOVA para as variáveis dos modelos de estudo do Grupo 2 ( $n=19$ ), nas três fases estudadas (T1, T2 e T3). Na presença de um resultado significativo, realizou-se o teste de Tukey (letras diferentes demonstram diferença significativa entre as medições).

\begin{tabular}{ccccc} 
& Inicial (T1) & Final (T2) & $\begin{array}{c}\text { Pós- } \\
\text { contenção (T3) }\end{array}$ & p \\
\cline { 2 - 4 } Variáveis & média (d.p.) & média (d.p.) & média (d.p.) & \\
\cline { 2 - 4 } LITTLE & $6,35(2,67)^{\mathrm{A}}$ & $0,00(0,00)^{\mathrm{B}}$ & $0,80(0,76)^{\mathrm{C}}$ & $0,000^{*}$ \\
INTERC & $33,35(2,71)^{\mathrm{A}}$ & $34,42(1,86)^{\mathrm{B}}$ & $34,24(2,06)^{\mathrm{AB}}$ & $0,038^{*}$ \\
INTERPB & $32,08(1,98)^{\mathrm{A}}$ & $35,16(1,76)^{\mathrm{B}}$ & $34,69(2,17)^{\mathrm{B}}$ & $0,000^{*}$ \\
INTERPB' & $37,32(2,51)^{\mathrm{A}}$ & $39,93(2,34)^{\mathrm{B}}$ & $39,23(2,07)^{\mathrm{B}}$ & $0,000^{*}$ \\
INTERMOL & $49,71(2,66)^{\mathrm{A}}$ & $50,61(2,04)^{\mathrm{A}}$ & $50,72(2,88)^{\mathrm{A}}$ & 0,104 \\
\cline { 1 - 4 } COMPR & $68,59(3,46)^{\mathrm{A}}$ & $71,21(3,75)^{\mathrm{B}}$ & $70,06(3,19)^{\mathrm{B}}$ & $0,000^{*}$
\end{tabular}

* Estatisticamente significativo para $\mathrm{p}<0,05$. 
observadas diferenças estatisticamente significativas entre os Grupos 1 e 2 (Tab. 11).

A Tabela 12 mostra os resultados dos testes de correlação de Pearson. Observou-se uma correlação

TABELA 6 - Resultados do teste $t$ independente, aplicado às variáveis estudadas, para verificar as diferenças na fase inicial (T1), entre os Grupos 1 e 2.

\begin{tabular}{|c|c|c|c|c|c|}
\hline \multirow{2}{*}{ Variáveis } & \multicolumn{2}{|c|}{$\begin{array}{c}\text { Grupo } 1 \text { (Classe I) } \\
\qquad(n=19)\end{array}$} & \multicolumn{2}{|c|}{$\begin{array}{c}\text { Grupo } 2 \text { (Classe II) } \\
\text { (n=19) }\end{array}$} & \multirow{2}{*}{$\mathbf{p}$} \\
\hline & média & d.p. & média & d.p. & \\
\hline LITTLE1 & 7,83 & 3,14 & 6,35 & 2,67 & 0,126 \\
\hline INTERC1 & 33,79 & 2,36 & 33,35 & 2,71 & 0,603 \\
\hline INTERPB1 & 32,62 & 1,91 & 32,08 & 1,98 & 0,401 \\
\hline INTERPB'1 & 37,91 & 2,94 & 37,32 & 2,51 & 0,514 \\
\hline INTERMOL1 & 49,49 & 3,16 & 49,71 & 2,66 & 0,820 \\
\hline COMPR1 & 68,33 & 4,72 & 68,59 & 3,46 & 0,849 \\
\hline
\end{tabular}

TABELA 7 - Resultados do teste $t$ independente, aplicado às variáveis estudadas, para verificar as diferenças na fase pós-tratamento (T2), entre os Grupos 1 e 2

\begin{tabular}{cccccc} 
& \multicolumn{2}{c}{$\begin{array}{c}\text { Grupo } 1 \text { (Classe I) } \\
\text { Variáveis }\end{array}$} & \multicolumn{2}{c}{$\begin{array}{c}\text { Grupo } 2 \text { (Classe II) } \\
(\mathbf{n}=19)\end{array}$} & p \\
\cline { 2 - 5 } & média & d.p. & média & d.p. & \\
LITTLE2 & 0,34 & 0,68 & 0,00 & 0,00 & $0,034^{*}$ \\
\hline INTERC2 & 34,46 & 1,48 & 34,42 & 1,86 & 0,943 \\
INTERPB2 & 35,91 & 1,63 & 35,16 & 1,76 & 0,182 \\
\hline INTERPB'2 & 40,90 & 2,19 & 39,93 & 2,34 & 0,198 \\
INTERMOL2 & 51,53 & 2,86 & 50,61 & 3,04 & 0,347 \\
COMPR2 & 71,01 & 3,45 & 71,21 & 3,75 & 0,868
\end{tabular}

* Estatisticamente significativo para $p<0,05$.

TABELA 8 - Resultados do teste $t$ independente, aplicado às variáveis estudadas, para verificar as diferenças no estágio pós-contenção (T3) entre os grupos 1 e 2 .

\begin{tabular}{cccccc}
\multirow{2}{*}{ Variáveis } & \multicolumn{2}{c}{$\begin{array}{c}\text { Grupo 1 (Classe I) } \\
(\mathbf{n = 1 9 )}\end{array}$} & \multicolumn{2}{c}{$\begin{array}{c}\text { Grupo 2 (Classe II) } \\
\text { (n=19) }\end{array}$} & p \\
\cline { 2 - 5 } & Média & d.p. & Média & d.p. & \\
LITTLE3 & 2,01 & 1,87 & 0,80 & 0,76 & $0,012^{*}$ \\
INTERC3 & 34,29 & 1,47 & 34,24 & 2,06 & 0,939 \\
\hline INTERPB3 & 34,66 & 1,54 & 34,69 & 2,17 & 0,958 \\
\hline INTERPB'3 & 40,02 & 2,04 & 39,23 & 2,07 & 0,246 \\
INTERMOL3 & 51,34 & 2,69 & 50,72 & 2,88 & 0,498 \\
\hline COMPR3 & 69,48 & 3,38 & 70,06 & 3,19 & 0,591 \\
\hline
\end{tabular}

negativa, estatisticamente significativa, entre a quantidade de recidiva do apinhamento anterossuperior e a quantidade de redução da distância entre os primeiros molares (Tab. 12).

TABELA 9 - Resultados do teste $t$ independente, aplicado às variáveis estudadas, para verificar as diferenças das alterações do tratamento (T2-T1), entre os grupos 1 e 2 .

\begin{tabular}{cccccc} 
& \multicolumn{2}{c}{$\begin{array}{c}\text { Grupo } 1 \text { (Classe I) } \\
\text { Variáveis }\end{array}$} & \multicolumn{2}{c}{$\begin{array}{c}\text { Grupo 2 (Classe II) } \\
(\mathbf{n = 1 9 )}\end{array}$} & \multicolumn{2}{c}{ p } \\
\cline { 2 - 5 } & média & d.p. & média & d.p. & \\
\cline { 2 - 5 } LITTLE2-1 & $-7,48$ & 3,24 & $-6,35$ & 2,67 & 0,247 \\
\hline INTERC2-1 & 0,56 & 2,67 & 1,06 & 2,07 & 0,525 \\
\hline INTERPB2-1 & 3,29 & 1,78 & 3,08 & 1,88 & 0,723 \\
INTERPB'2-1 & 2,98 & 2,27 & 2,60 & 2,69 & 0,641 \\
\hline INTERM0L2-1 & 2,03 & 2,13 & 0,90 & 2,78 & 0,169 \\
\hline COMPR2-1 & 2,68 & 3,18 & 2,61 & 3,01 & 0,951
\end{tabular}

TABELA 10 - Resultados do teste $t$ independente, aplicado às variáveis estudadas, para verificar as diferenças das alterações no período pós-tratamento (T3-T2), entre os grupos 1 e 2.

\begin{tabular}{|c|c|c|c|c|c|}
\hline \multirow{2}{*}{ Variáveis } & \multicolumn{2}{|c|}{$\begin{array}{c}\text { Grupo } 1 \text { - Classe I } \\
\qquad(n=19)\end{array}$} & \multicolumn{2}{|c|}{$\begin{array}{c}\text { Grupo } 2 \text { (Classe II) } \\
\qquad(n=19)\end{array}$} & \multirow{2}{*}{$\mathbf{p}$} \\
\hline & média & d.p. & média & d.p. & \\
\hline LITTLE3-2 & 1,67 & 1,45 & 0,80 & 0,76 & $0,026^{*}$ \\
\hline INTERC3-2 & $-0,16$ & 0,89 & $-0,17$ & 0,77 & 0,984 \\
\hline INTERPB3-2 & $-1,25$ & 1,10 & $-0,47$ & 1,47 & 0,072 \\
\hline INTERPB'3-2 & $-0,85$ & 0,97 & $-0,70$ & 1,21 & 0,670 \\
\hline INTERMOL3-2 & $-0,18$ & 0,90 & 0,10 & 0,99 & 0,348 \\
\hline COMPR3-2 & $-1,53$ & 0,75 & $-1,15$ & 1,39 & 0,289 \\
\hline
\end{tabular}

* Estatisticamente significativo para $p<0,05$.

TABELA 11 - Resultados do teste $t$ independente, aplicado às variáveis estudadas, para verificar as diferenças das alterações no período total de observação (T3-T1), entre os grupos 1 e 2.

\begin{tabular}{cccccc} 
& \multicolumn{2}{c}{$\begin{array}{c}\text { Grupo } 1 \text { (Classe I) } \\
\text { Variáveis }\end{array}$} & \multicolumn{2}{c}{$\begin{array}{c}\text { Grupo 2 (Classe II) } \\
(\mathbf{n = 1 9 )}\end{array}$} & p \\
\cline { 2 - 5 } & Média & d.p. & Média & d.p. & \\
LITTLE3-1 & $-5,81$ & 3,94 & $-5,55$ & 2,34 & 0,804 \\
\hline INTERC3-1 & 0,50 & 2,03 & 0,89 & 2,34 & 0,588 \\
\hline INTERPB3-1 & 2,04 & 1,56 & 2,61 & 2,24 & 0,370 \\
\hline INTERPB'3-1 & 2,10 & 2,20 & 1,90 & 2,13 & 0,775 \\
\hline INTERMOL3-1 & 1,84 & 2,34 & 1,01 & 2,42 & 0,287 \\
\hline COMPR3-1 & 1,14 & 3,10 & 1,46 & 2,29 & 0,718 \\
\hline
\end{tabular}

* Estatisticamente significativo para $p<0,05$. 
TABELA 12 - Resultados do teste de correlação de Pearson.

$\begin{array}{ccc}\text { Variáveis } & \mathbf{r} & \mathbf{p} \\ \text { LITTLE1 x LITTLE3 } & 0,101 & 0,545 \\ \text { LITTLE1 x LITTLE3-2 } & 0,104 & 0,533 \\ \text { LITTLE2-1 x LITTLE3-2 } & -0,021 & 0,899 \\ \text { LITTLE3-2 } \text { x INTERC3-2 } & 0,128 & 0,441 \\ \text { LITTLE3-2 x INTERPB3-2 } & -0,296 & 0,071 \\ \text { LITTLE3-2 x INTERPB'3-2 } & -0,177 & 0,286 \\ \text { LITTLE3-2 x INTERM0L3-2 } & -0,342 & 0,035^{*} \\ \text { LITTLE3-2 } \text { x COMPR3-2 } & -0,301 & 0,065\end{array}$

* Estatisticamente significativo para $p<0,05$.

\section{DISCUSSÃO}

Apesar da extensa literatura relacionada ao estudo da estabilidade no alinhamento dentário anteroinferior ${ }^{3,4,11,17,18,20,30}$, os estudos que avaliaram o comportamento dos incisivos superiores após a remoção das contenções apresentam-se em menor número $8,9,14,23,29$. Possivelmente, esse fato se justifique pela maior frequência de recidiva no segmento anteroinferior ${ }^{27,31}$. Apesar da recidiva anterossuperior apresentar-se menos prevalente, a busca por fatores que possam auxiliar na estabilidade no segmento anterossuperior apresenta validade, já que uma deterioração do tratamento nesse segmento da arcada também pode resultar em um comprometimento estético e funcional. Devido à sua localização na arcada, o apinhamento na região anterossuperior tende a apresentar-se mais visível e, consequentemente, promover maiores prejuízos estéticos do que a irregularidade dentária anteroinferior. Entretanto, deve-se ter consciência de que, dependendo da altura do sorriso do paciente, pode-se observar uma inversão dessa situação ${ }^{5}$.

Para a obtenção da amostra, foram selecionados os modelos de estudo de grupos de pacientes que apresentavam relação molar de Classe I (Grupo 1) e relação molar de, no mínimo, três quartos de Classe II (Grupo 2), tratados sem extrações. Outro critério de seleção da amostra foi que todos os pacientes tivessem sido tratados com a mecânica ortodôntica fixa, tipo Edgewise, nas arcadas superior e inferior.
A seleção da amostra foi realizada de forma a eliminar o maior número possivel de fatores que pudessem influenciar nos resultados. Dessa forma, uma das maiores preocupações na condução desse trabalho consistiu na obtenção de grupos compatíveis, o que viabilizaria uma correta comparação e, consequentemente, favoreceria a interpretação e a confiabilidade dos resultados. Para tanto, realizou-se uma homogeneização das características ao início e ao término do tratamento ortodôntico. Os grupos foram compatibilizados quanto ao protocolo de tratamento, o tipo de acessório ortodôntico utilizado, em relação à distribuição dos sexos e da má oclusão inicial. Além disso, foram, também, compatibilizadas características como: as idades inicial e final, o tempo de tratamento e o tempo de avaliação pós-contenção. Dessa maneira, as alterações que ocorreram no período pós-contenção puderam ser analisadas com maior segurança.

Em relação à estabilidade, notam-se divergências na literatura sobre o comportamento pós-contenção nos diferentes tipos de má oclusão (Classe I e Classe II ${ }^{25,31}$. Além disso, alguns estudos relatam que quanto maior a severidade inicial da má oclusão, maior a recidiva e menor a estabilidade em longo prazo $^{10,18}$. Sendo assim, optou-se por investigar a estabilidade do alinhamento dentário anterossuperior, no período pós-contenção, em dois grupos que apresentavam características similares em relação à quantidade inicial de apinhamento anterossuperior. Com o objetivo de verificar a compatibilidade intergrupos em relação à quantidade de apinhamento inicial, realizou-se o teste t para a variável índice de irregularidade de Little inicial. Os resultados demonstraram que não existiam diferenças estatisticamente significativas entre os grupos nas quantidades de apinhamento anterossuperior pré-tratamento (Tab. 3).

\section{Comparação intragrupos}

Os resultados do ANOVA e do teste de Tukey, para o Grupo 1 (Tab. 4), demonstraram alterações estatisticamente significativas no índice de irregularidade de Little para a fase inicial em relação às fases final e pós-tratamento, e não demonstraram uma 
diferença estatisticamente significativa entre as fases final e pós-tratamento. Interpretando esses resultados, pode-se afirmar que o tratamento ortodôntico resultou em significativa redução do apinhamento dentário anterossuperior e, posteriormente, durante a fase pós-contenção, não se observou um aumento significativo dessa irregularidade.

A distância intercaninos não mostrou alteração significativa entre nenhuma das três fases avaliadas (Tab. 4). Esses resultados poderiam suportar o conceito de manutenção da distância intercaninos original no tratamento ortodôntico, pois ela tende a retornar aos valores iniciais, conforme já descrito por diversos autores ${ }^{12,28}$, que também concluíram que a expansão da distância intercaninos inferiores pode levar a uma deficiência nos resultados. Porém, fica difícil a distinção entre a recidiva da distância intercaninos e a diminuição dessa distância em função do passar dos anos (em oclusões normais), conforme estudaram e afirmaram outros autores ${ }^{18,28}$.

Em relação às alterações na dimensão e forma da arcada superior durante o tratamento, foram notados incrementos transversais significativos (aumento nas variáveis INTERPB, INTERPB', INTERMOL) e aumento também significativo no comprimento da arcada. No entanto, apenas a distância interprimeiros pré-molares sofreu uma diminuição na fase pós-contenção, no entanto, não atingindo os valores iniciais. $\mathrm{O}$ comprimento da arcada também apresentou uma alteração significativa no período pós-tratamento, atingindo valores próximos aos iniciais.

Os resultados do ANOVA e do teste de Tukey para o Grupo 2 (Tab. 5) demonstraram alterações estatisticamente significativas no índice de irregularidade de Little nos três tempos avaliados. Dessa forma, observou-se no Grupo 2 um comportamento diferente do Grupo 1 em relação às alterações da variável LITTLE, ou seja, uma redução significativa do apinhamento dentário anterossuperior durante o tratamento; e, posteriormente, durante o periodo pós-tratamento, uma recidiva significativa da irregularidade dos incisivos superiores, porém, não atingindo os valores iniciais.
As variáveis INTERC, INTERPB, INTERPB' e COMPR mostraram um aumento estatisticamente significativo da fase inicial para a fase final e não mostraram recidiva significativa quando comparadas as fases final e pós-contenção, praticamente mantendo os valores obtidos com o tratamento. Já a variável INTERMOL não mostrou alteração estatisticamente significativa em nenhuma das fases estudadas.

\section{Comparação intergrupos}

Quando comparados os Grupos 1 e 2, ou seja Classe I e Classe II de Angle, na fase inicial de tratamento, observou-se que nenhuma das variáveis apresentou diferença significativa entre os grupos (Tab. 6). Alguns trabalhos que avaliaram a recidiva do apinhamento na fase pós-contenção citam que a quantidade de apinhamento inicial está diretamente relacionada com a quantidade de recidiva ${ }^{1}$, muito embora outros autores $^{20}$ não tenham observado essa relação.

Em relação às dimensões da arcada superior, nota-se que o Grupo 1 e o Grupo 2 apresentavam dimensões transversais semelhantes entre si (Tab. 6). McNamara ${ }^{22}$ comentou que, geralmente, uma arcada superior que apresenta uma distância transpalatina de 36 a $39 \mathrm{~mm}$ é capaz de acomodar uma dentição permanente de tamanho médio, sem apinhamento ou espaçamento.

Conforme citado anteriormente, a severidade do apinhamento inicial e as medidas das demais variáveis foram semelhantes entre os dois grupos, permitindo uma maior confiabilidade dos resultados da comparação entre eles.

Quando comparados os Grupos 1 e 2, Classe I e Classe II de Angle, respectivamente, a única variável que apresentou diferença significativa entre os dois grupos foi o índice de irregularidade de Little, que se mostrou maior no grupo Classe I, evidenciando que o Grupo 2 teve um término de tratamento ligeiramente melhor que o Grupo 1 (Tab. 7). Embora estatisticamente significativa, a diferença entre os dois grupos foi de apenas $0,34 \mathrm{~mm}$, 
a qual pode ser considerada não significativa clinicamente. Little ${ }^{18}$ considerou valores entre $0 \mathrm{e}$ $1 \mathrm{~mm}$ como sendo os de um alinhamento ideal.

Ao final da fase pós-contenção, quando comparados os Grupos 1 e 2, a única variável que apresentou diferença estatisticamente significativa foi o indice de irregularidade de Little (LITTLE3), já as demais variáveis (INTERC3, INTERPB3, INTERPB'3, INTERMOL3 e COMPR3) não mostraram diferença estatisticamente significativa (Tab. 8). Esses resultados sugeriram um comportamento parecido dos grupos no transcorrer da fase pós-contenção, uma vez que ambos os grupos apresentaram, tanto ao final do tratamento quanto ao final do período pós-contenção, características bastante semelhantes.

A comparação dos Grupos 1 e 2 revelou que não houve diferença significativa entre os dois grupos em relação à quantidade de correção do apinhamento anterossuperior (LITTLE2-1, Tab. 9). Esse resultado era esperado, já que não existiam diferenças estatisticamente significativas entre os grupos nas quantidades de apinhamento inicial e final.

A redução da irregularidade dos incisivos superiores durante o tratamento foi similar à de estudos anteriores que avaliaram casos tratados sem extrações dentárias ${ }^{7,32}$. No entanto, alguns estudos tiveram uma redução menor dessa irregularidade durante o tratamento, devido ao fato de que o apinhamento inicial se apresentava menor ${ }^{8}$.

$O$ fato é que em todos esses estudos tentou-se zerar a irregularidade dos incisivos durante o tratamento. Sendo assim, a variação na quantidade de correção se deu devido à variação na severidade do apinhamento inicial de cada caso.

Os Grupos 1 e 2 demonstraram que as quantidades de recidiva da variável LITLLE3-2 que ocorreu neles foram diferentes estatisticamente: o Grupo 1 (Classe I de Angle) apresentou uma recidiva média de $1,67 \mathrm{~mm}( \pm 1,45 \mathrm{~mm})$, ao passo que o Grupo 2 (Classe II de Angle) mostrou uma recidiva média do apinhamento de $0,80 \mathrm{~mm}( \pm 0,76 \mathrm{~mm})$, evidenciando uma maior estabilidade do tratamento no Grupo 2 (Tab. 10).
Essa diferença significativa entre a recidiva do Grupo Classe I e do Grupo Classe II pode ser devida ao fato de que, no Grupo Classe II, tratado sem extração, houve imprescindivelmente a necessidade de distalização dos molares superiores e, portanto, um maior espaço para o nivelamento dos incisivos superiores, que se encontravam apinhados. Essa distalização dos molares, com consequente ganho de espaço para o nivelamento dos incisivos, pode ter favorecido a estabilidade. No caso do Grupo Classe I, os molares superiores permaneceram em suas posições iniciais e o apinhamento teve que ser resolvido por protrusão dos incisivos superiores, talvez causando uma maior recidiva do apinhamento anterossuperior nesse grupo.

Sadowsky et al. ${ }^{27}$, avaliando a estabilidade de casos tratados sem extrações, observaram uma recidiva, após 5 anos da remoção das contenções, relativamente similar $(1,1 \mathrm{~mm})$. Entretanto, Moussa, O'Reilly e Close ${ }^{23}$ observaram, 8 a 10 anos após a remoção das contenções, resultados mais favoráveis em relação a essa recidiva. Vaden et $\mathrm{al} .{ }^{32}$ observaram que $96 \%$ da correção do apinhamento anterossuperior durante o tratamento foram mantidos após 15 anos do término do tratamento. A quantidade de apinhamento aumentou de $1,5 \mathrm{~mm}$, ao final do tratamento, para $1,8 \mathrm{~mm}$ na avaliação pós-contenção. Ferris et al. ${ }^{9}$, também avaliando a recidiva desse segmento da arcada em casos tratados sem extrações, observaram durante a fase pós-contenção (7,9 anos) um aumento na irregularidade dos incisivos superiores de apenas $0,47( \pm 1,19)$. A maior estabilidade do alinhamento dos dentes anterossuperiores nesses trabalhos talvez possa ser explicada pelo prolongamento do tempo de uso das contenções após o tratamento ortodôntico fixo $3,23,27$. No trabalho realizado por Sadowsky et al. ${ }^{27}$, o tempo médio de uso da contenção foi de 8,4 anos. O trabalho realizado por Moussa, O'Reilly e Close ${ }^{23}$ apresentou um tempo de contenção para a arcada inferior de 6,6 anos, com uso contínuo de uma placa de Hawley na arcada superior por 2 anos. Na pesquisa realizada por Vaden et al. ${ }^{32}$, os pacientes utilizaram placas de Hawley nas arcadas superior e inferior ou uma placa de Hawley 
na arcada superior e uma contenção fixa $(3 \times 3)$ na arcada inferior. $\mathrm{O}$ primeiro controle pós-tratamento foi realizado somente após 6 anos. O estudo realizado por Ferris et al. ${ }^{9}$, apresentou um protocolo de contenção que incluía o uso de contenção removível na arcada superior por 3 anos ( 1 ano de uso contínuo) e o uso de $3 \times 3$ ou placa de Hawley na arcada inferior por um período médio de 3 anos. No presente trabalho, todos os pacientes receberam como contenção uma placa de Hawley na arcada superior por um período médio de 1 ano e um fio de aço colado de canino a canino na arcada inferior $(3 \times 3)$ por um período de 3 anos.

Erdinc, Nanda e Isiksal ${ }^{8}$ propuseram a avaliação em longo prazo do apinhamento dos incisivos superiores e inferiores em pacientes ortodônticos tratados com e sem extrações de pré-molares. Observaram, 4 anos e 11 meses pós-contenção, aumentos na irregularidade dos incisivos superiores de $0,19 \mathrm{~mm}$ e $0,12 \mathrm{~mm}$ em casos tratados, respectivamente, com e sem extrações. O grupo tratado com extração apresentava um apinhamento inicial de $4,4 \mathrm{~mm}$, enquanto o grupo tratado sem extrações apresentava um apinhamento inicial de apenas $1,94 \mathrm{~mm}$. As contenções superior e inferior (placas de Hawley) foram removidas pelo menos dois anos antes da avaliação pós-contenção. A excepcional estabilidade desse trabalho pode estar relacionada à pequena quantidade de apinhamento inicial e a um curto intervalo entre a remoção das contenções e a avaliação pós-contenção.

Surbeck et al. ${ }^{29}$ observaram uma influência direta da quantidade de apinhamento anterossuperior inicial sobre a quantidade de recidiva pós-contenção e, dessa forma, sugeriram o emprego de diferentes protocolos de contenção e o esclarecimento aos pacientes, de forma individualizada, quanto à possibilidade de instabilidade pós-contenção, de acordo com a irregularidade inicial.

Entretanto, analisando-se os resultados de outros estudos e os resultados obtidos nesse, parece improvável uma correlação positiva entre a quantidade de apinhamento anterossuperior inicial e a quantidade de recidiva pós-contenção. Por exemplo, no presente trabalho, os Grupos 1 e 2, respectivamente, apresentavam, ao início do tratamento, $7,83 \mathrm{~mm}( \pm 3,14)$ e $6,35 \mathrm{~mm}( \pm 2,67)$, em relação à quantidade de apinhamento inicial; e uma recidiva pós-contenção média de $1,67 \mathrm{~mm}( \pm 1,45 \mathrm{~mm})$ para o Grupo $1 \mathrm{e}$ $0,80 \mathrm{~mm}( \pm 0,76 \mathrm{~mm})$ para o Grupo 2. A quantidade média de recidiva do Grupo 1 nesse trabalho apresentou-se maior do que a verificada por Ferris et al. ${ }^{9}$, Sadowsky et al. ${ }^{27}$ e Vaden et al. ${ }^{32}$, que constataram maiores valores para a irregularidade inicial dos incisivos superiores: $10,45 \mathrm{~mm}, 8,0 \mathrm{~mm}$ e $7,9 \mathrm{~mm}$, respectivamente. Esses trabalhos, mesmo apresentando quantidade de apinhamento inicial ligeiramente maior do que a do presente estudo, demonstraram uma menor quantidade de recidiva do apinhamento no período pós-contenção $(0,47 \mathrm{~mm} ; 1,1 \mathrm{~mm}$ e $0,3 \mathrm{~mm}$, respectivamente).

Em relação às alterações totais, o índice de irregularidade dos incisivos superiores apresentou uma redução de $5,81 \mathrm{~mm}( \pm 3,94 \mathrm{~mm})$ e $5,55 \mathrm{~mm}( \pm 2,34 \mathrm{~mm})$ para os Grupos 1 e 2, respectivamente, e não foram observadas diferenças estatisticamente significativas entre os grupos (Tab. 11).

\section{Correlação}

Testes de correlação foram realizados na amostra total para obtenção dos coeficientes de correlação de Pearson. Dessa forma, verificou-se a existência ou não de correlação entre a irregularidade dos incisivos na fase inicial (LITTLE1) e a irregularidade no estágio pós-contenção (LITTLE3); entre o apinhamento inicial (LITTLEl) e a quantidade de recidiva do apinhamento anterossuperior no período pós-contenção (LITTLE3-2); e entre a quantidade de correção do apinhamento durante o tratamento (LITTLE2-1) e a quantidade de recidiva após a remoção das contenções (LITTLE3-2). Buscou-se, também, verificar uma possível correlação entre a recidiva do apinhamento anterossuperior (LITTLE3-2) e as variáveis que refletem as alterações em todas as dimensões da arcada superior no período pós-contenção (INTERC3-2, INTERPB3-2, INTERPB'3-2, INTERMOL3-2 e COMPR3-2). Os resultados obtidos encontram-se na Tabela 12. 
Os testes de correlação apresentaram, em sua maioria, resultados não significativos. Observou-se que a quantidade de apinhamento inicial não influenciou na recidiva pós-contenção, como já descrito em estudos prévios ${ }^{2,3,20}$. Surbeck et al..$^{29}$, em contrapartida, relataram uma correlação positiva entre a quantidade de irregularidade dentária anterossuperior e a quantidade de recidiva nesse segmento da arcada. Afirmaram que a tendência de se observar uma recidiva na irregularidade dos incisivos superiores aumenta 2,3 vezes para cada $0,2 \mathrm{~mm}$ de deslocamento dos pontos de contato dos incisivos em relação à arcada dentária. Além disso, a cada $4^{\circ}$ de rotação dos incisivos ao início do tratamento, tem-se um aumento de 2,7 vezes na probabilidade de recidiva da irregularidade. Os autores também ressaltaram que dentes parcialmente alinhados durante o tratamento também apresentam risco significativo de recidiva.

A quantidade de recidiva do apinhamento anterossuperior no período pós-contenção (LITTLE3-2) apresentou correlação estatisticamente significativa $(\mathrm{p}<0,035)$ com as alterações da distância intermolares (INTERMOL3-2) durante o mesmo período. A correlação observada apresenta coeficiente de valor negativo. Interpretando o resultado, verifica-se que quanto maior a redução da distância intermolares na fase pós-contenção, maior a recidiva do apinhamento anterossuperior.
Embora essa correlação apresente significância estatística, o valor de seu coeficiente revela uma correlação fraca (valor de $r=-0,342$ ). Portanto, pode-se afirmar que a correlação observada entre a recidiva do apinhamento anterossuperior e a redução desta distância apresenta pouca significância clínica. Essa diminuição da distância intermolares provavelmente se dá devido a esses dentes estarem com um nível de rotação relativamente alto ao início do tratamento e, ao serem observados no período pós-contenção, apresentarem uma recidiva dessa rotação, o que pode explicar, em parte, a diminuição da distância intermolares nessa fase.

\section{CONCLUSÕES}

De acordo com a amostra e a metodologia utilizada e a partir dos resultados expostos e discutidos, pode-se concluir que:

» Houve uma maior recidiva, estatisticamente significativa, do apinhamento anterossuperior no Grupo Classe I de Angle, quando comparado com o Grupo Classe II de Angle.

"As variáveis estudadas mostraram aumento com o tratamento da má oclusão, porém, apresentaram ligeira recidiva no período pós-contenção. A única variável que mostrou correlação com a recidiva do apinhamento anterossuperior foi a redução da distância intermolares no período pós-contenção.

\title{
Relapse of maxillary anterior crowding in Class I and Class II malocclusions orthodontically treated without extractions
}

\begin{abstract}
Objective: The present study aimed to retrospectively compare the postretention stability of maxillary anterior incisors alignment in Class I and Class II patients. Methods: The sample comprised 38 patients of both genders, treated with nonextraction and Edgewise mechanics, divided into two groups: Group 1, comprised of 19 patients, at a mean age of 13.06 years, with Class I malocclusion and initial maxillary anterior crowding greater than $3 \mathrm{~mm}$; Group 2, comprised of 19 patients, at a mean age of 12.54 years, with Class II malocclusion, and also with a initial maxillary anterior crowding greater than $3 \mathrm{~mm}$. In the dental casts of pretreatment, posttreatment and postretention phase, the Little's irregularity index, intercanine distance and distance between first and between second premolars, intermolar distance and maxillary arch length were measured. For intragroup comparison among the three times of evaluation, it was used the one-way ANOVA followed by Tukey test. Intergroup comparison was performed by independent $t$ tests. To verify the presence of correlation, the Pearson correlation test was used. Results: It was evidenced greater stability of treatment in Group 2 (Class II), because during the postretention period it was observed a softer relapse of maxillary anterior crowding in Group $2(0.80 \mathrm{~mm})$ than in Group $1(1.67 \mathrm{~mm})$. Conclusion: It can be concluded that the treatment of maxillary anterior crowding is more stable in Class II malocclusion than in Class I malocclusion.
\end{abstract}

Keywords: Maxillary anterior crowding. Posttreatment stability. 


\section{REFERÊNCIAS}

1. Artun J, Garol JD, Little RM. Long-term stability of mandibular incisors following successful treatment of Class II, division 1, malocclusions. Angle Orthod. 1996;66(3):229-38.

2. Artun J, Krogstad O, Little RM. Stability of mandibular incisors following excessive proclination: a study in adults with surgically treated mandibular prognathism. Angle Orthod. 1990;60(2):99-106.

3. Azizi M, Shrout MK, Haas AJ, Russell CM, Hamilton EH Jr. A retrospective study of Angle Class I malocclusions treated orthodontically without extractions using two palatal expansion methods. Am J Orthod Dentofacial Orthop. 1999;116(1):101-7.

4. Blake M, Bibby K. Retention and stability: a review of the literature. Am J Orthod Dentofacial Orthop. 1998;114(3):299-306

5. Canuto LFG. Avaliação da influência da realização da expansão rápida da maxila sobre a recidiva do apinhamento ântero-superior, em casos tratados ortodonticamente sem extrações [dissertação]. Bauru (SP): Universidade de São Paulo; 2006

6. Dahlberg G. Statistical methods for medical and biological students. New York: Interscience; 1940

7. Destang DL, Kerr WJ. Maxillary retention: is longer better? Eur J Orthod. 2003 Feb;25(1):65-9.

8. Erdinc AE, Nanda RS, Isiksal E. Relapse of anterior crowding in patients treated with extraction and nonextraction of premolars. Am J Orthod Dentofacial Orthop. 2006;129(6):775-84.

9. Ferris T, Alexander RG, Boley J, Buschang PH. Long-term stability of combined rapid palatal expansion-lip bumper therapy followed by full fixed appliances. Am J Orthod Dentofacial Orthop. 2005 Sep;128(3):310-25

10. Freitas KM, Janson G, Freitas MR, Pinzan A, Henriques JF, Pinzan-Vercelino CR. Influence of the quality of the finished occlusion on postretention occlusal relapse. Am J Orthod Dentofacial Orthop. 2007 Oct;132(4):428.e9-14.

11. Freitas KM, de Freitas MR, Henriques JF. Postretention relapse of mandibular anterior crowding in patients treated without mandibular premolar extraction. Am J Orthod Dentofacial Orthop. 2004;125(4):480-7.

12. Glenn G, Sinclair PM, Alexander RG. Nonextraction orthodontic therapy: posttreatment dental and skeletal stability. Am J Orthod Dentofacial Orthop. 1987 Oct;92(4):321-8

13. Houston WJB. The analysis of errors in orthodontic measurements. Am J Orthod. 1983 May;83(5):382-90.

14. Huang $L$, Artun J. Is the postretention relapse of maxillary and mandibular incisor alignment related? Am J Orthod Dentofacial Orthop. 2001;120(1):9-19.

15. Kahl-Nieke B, Fischbach $\mathrm{H}$, Schwarze CW. Post-retention crowding and incisor irregularity: a long-term followup evaluation of stability and relapse. $\mathrm{Br} \mathrm{J}$ Orthod. 1995;22(3):249-57.
16. Little RM. The irregularity index: a quantitative score of mandibular anterior alignment. Am J Orthod. 1975;68(5):554-63.

17. Little RM. Stability and relapse of dental arch alignment. Br J Orthod. 1990;17(3):235-41.

18. Little RM. Stability and relapse of mandibular anterior alignment: University of Washington studies. Semin Orthod. 1999;5(3):191-204

19. Little RM, Riedel RA, Stein A. Mandibular arch length increase during the mixed dentition: postretention evaluation of stability and relapse. Am J Orthod Dentofacial Orthop. 1990;97(5):393-404.

20. Little RM, Wallen TR, Riedel RA. Stability and relapse of mandibular anterior alignment - first premolar extraction cases treated by traditional Edgewise orthodontics. Am J Orthod. 1981 Oct;80(4):349-65.

21. Martins PP. Avaliação da influência da expansão rápida da maxila sobre a recidiva do apinhamento ântero-superior em casos tratados ortodonticamente com extrações de pré-molares [dissertação]. Bauru (SP): Universidade de São Paulo; 2007.

22. McNamara JA. Maxillary transverse deficiency. Am J Orthod Dentofacial Orthop. 2000;117(5):567-70.

23. Moussa R, O'Reilly MT, Close JM. Long-term stability of rapid palatal expander treatment and Edgewise mechanotherapy. Am J Orthod Dentofacial Orthop. 1995;108(5):478-88.

24. Naraghi S, Andrén A, Kjellberg H, Mohlin BO. Relapse tendency after orthodontic correction of upper front teeth retained with a bonded retainer. Angle Orthod. 2006;76(4):570-6.

25. Ormiston JP, Huang GJ, Little RM, Decker JD, Seuk GD. Retrospective analysis of long-term stable and unstable orthodontic treatment outcomes. Am J Orthod Dentofacia Orthop. 2005;128(5):568-74; quiz 669.

26. Sadowsky C, Sakols El. Long-term assessment of orthodontic relapse. Am J Orthod. 1982;82(6):456-63.

27. Sadowsky C, Schneider BJ, BeGole EA, Tahir E. Long-term stability after orthodontic treatment: nonextraction with prolonged retention. Am J Orthod Dentofacial Orthop 1994; 106(3):243-9.

28. Sinclair PM, Little RM. Maturation of untreated normal occlusions. Am J Orthod. 1983;83(2):114-23.

29. Surbeck BT, Artun J, Hawkins NR, Leroux B. Associations between initial, posttreatment, and postretention alignment of maxillary anterior teeth. Am J Orthod Dentofacial Orthop. 1998;113(2):186-95.

30. Thilander B. Orthodontic relapse versus natural development. Am J Orthod Dentofacial Orthop. 2000;117(5):562-3.

31. Uhde MD, Sadowsky C, BeGole EA. Long-term stability of dental relationships after orthodontic treatment. Angle Orthod. 1983;53(3):240-52.

32. Vaden JL, Harris EF, Gardner RL. Relapse revisited. Am J Orthod Dentofacial Orthop. 1997;111(5):543-53. 